Vol. 67, N. ${ }^{\circ}$ IO6 (noviembre 2015), 37-52

\title{
«COMO CUALQUIER OTRO TRABAJO»: ORGANIZANDO A LAS TRABAJADORAS REMUNERADAS DEL HOGAR EN ECUADOR
}

\author{
ERYNN MASI DE CASANOVA \\ Universidad de Cincinnati, EE. UU. \\ Recepción manuscrito: 4 de agosto de 2015 \\ Aceptación versión final: 30 de octubre de 2015
}

\begin{abstract}
RESUMEN El trabajo remunerado del hogar (TRH) hace borrosa la distinción tradicional entre el trabajo productivo (que produce utilidades) y el trabajo reproductivo (reproducción social), lo cual influye el contexto en el cual se desarrollan movimientos laborales de TRH. El presente artículo presenta un estudio de caso considerando tres retos que enfrenta un movimiento social de TRH en Guayaquil, Ecuador. La organización investigada enfrenta estos retos con una gama de estrategias y actividades, entre ellas la redefinición del TRH como «trabajo regular» 0 un «trabajo como cualquier otro». ¿Habrá ventaja en usar la táctica retórica de igualdad con otros empleos para promover los derechos de las que desempeñan la reproducción social?
\end{abstract}

palabras Clave Trabajo doméstico, empleo femenino, movimientos laborales, movimientos sociales.

ABSTRACT Home remunerated work (TRH, for its acronym in Spanish) makes the distinction between productive work (the one producing income) and the reproductive work (the one involving social reproduction) blurry. This affects the context where the TRH working movements are developed. This current article reports a case study, considering three challenges that a TRH work social movement faces in Guayaquil, Ecuador. The organization that has been investigated is facing challenges with an array of strategies and activities, among them the redefinition of TRH as «regular work» or «a job as any other one». We wonder whether there is an advantage in using the rhetorical tactic of equality with other jobs to promote the rights for those women who perform the social reproduction.

KEYWORDS Household work, feminine job, working movements, social movements.

JEL CODES J16.

\section{INTRODUCCIÓN}

Este artículo tiene por objeto estudiar el proceso de organización de las trabajadoras remuneradas del hogar (TRH) en el Ecuador y conocer las dificultades que enfrentan; para ello se ha hecho un estudio de caso de una organización de trabajadoras con sede en Guayaquil para examinar la estrategia retórica de la redefinición del trabajo doméstico como un trabajo 
«regular», sin centrarse particularmente en las características y problemas que enfrentan las TRH, también denominadas trabajadoras domésticas. Al final del artículo, queda la cuestión de si se pierde algo cuando estas especiales condiciones de trabajo se equiparan con situaciones de empleo distintas de «otros trabajadores». ¿Habrá una ventaja estratégica en usar la táctica de igualdad con otros empleos en la movilización colectiva? ¿Tiene poder la comparación de la reproducción social con el trabajo productivo? El marco o contexto más grande para esta investigación es el rompecabezas de legislación sobre el trabajo doméstico remunerado en América Latina. Aunque se hayan aprobado leyes que protegen a las TRH en países como Argentina, Bolivia, Brasil, las trabajadoras del hogar en Ecuador todavía no tienen un marco jurídico. Tomando como estudio de caso a una organización ecuatoriana, se resaltan los retos prácticos que enfrentan los esfuerzos para organizar a las TRH y se analiza el discurso que emplea la organización. Para lograr no solo el reconocimiento legal, sino también para garantizar que las TRH puedan ejercer sus derechos, hay que recuperar o valorizar el trabajo de la reproducción social de alguna forma.

El trabajo remunerado del hogar es un aspecto constante de la vida urbana en América Latina. El arreglo informal de este empleo sigue existiendo por la necesidad que tienen las mujeres de bajos recursos de trabajar para mantenerse ellas y sus familias, y por la entrada de las mujeres de clase media a la fuerza laboral. Se han realizado estudios importantes sobre el trabajo remunerado del hogar en América Latina y el Caribe (Brites, 2014; Chaney y Garcia Castro, 1991; Goldsmith 2006; Gorbán, 2013; Gorbán y Tizziani, 2014; Karides, 2002; Pereyra, 2013), y en los Estados Unidos y otros países (Ehrenreich y Hochschild, 2004; HondagneuSotelo, 2007; Lan, 2006; Romero, 2002). Estos estudios investigan el trabajo del hogar en el contexto de la desigualdad socioeconómica, y examinan cómo este trabajo ayuda a mantener esta desigualdad. La literatura académica también se enfoca en los aspectos raciales/étnicos del trabajo remunerado del hogar. Algunos estudios han tratado la organización y movilización de las TRH, pero ninguno de estos incluye a Ecuador como sitio de investigación (Bernardino-Costa, 2011; Cabezas Fernández, 2012; Cornwall, Oliveira, y Gonçalvez, 2013; Karides, 2002; Maich, 2014; Menon, 2013; Mose Brown, 2011).

\section{EL TRABAJO REMUNERADO DEL HOGAR COMO TRABAJO INFORMAL}

El crecimiento de la economía informal en América Latina ha sido asociado con el incremento del trabajo informal, mal pagado, inestable, precario, de tiempo parcial, y temporal (Benería, 2003, p. 95). La fuerza laboral femenina se encuentra concentrada en la economía informal, y en Ecuador 63,7 por ciento del empleo femenino se caracteriza como informal (orT, 2013). El trabajo informal de las mujeres de las clases populares es estrategia de superviviencia más que una carrera o un vehículo para el ascenso socioeconómico (Benería, 2003, p. 112; Lautier, 2003). Estas mujeres muchas veces tienen «múltiples estrategias de ingresos» (Rothstein, 1995).

En el mundo, 43,6 millones de mujeres son TRH; este número representa el 7,5\% de la población femenina empleada (oIT, 2011). En América Latina, 14 millones de mujeres, equivalente al 14\% de las trabajadoras de la región, desempeñan trabajos pagados en domicilios (Estrada, 2009). En Ecuador, aproximadamente el 18\% de las mujeres que trabajan por 
remuneración son TRH: un total de 170.000 trabajadoras en el 2001, y 158.000 en 2012 (Reyes y Camacho, 2001; INEC, 2013). La mayoría de estas trabajadoras se encuentran en las áreas urbanas del país. El carácter informal del trabajo remunerado del hogar implica que estas figuras no captan las dimensiones exactas de esta fuerza laboral. Las mujeres de escasos recursos frecuentemente describen el trabajo remunerado del hogar como la opción de empleo menos atractiva, por el pago minúsculo y la posibilidad de explotación; muchas prefieren otros trabajos informales (Casanova, 2011).

Así que el sector informal es donde la mayoría de las TRH se ubican (Blofield, 2012). Sin embargo, si definimos al trabajo informal como una forma de empleo «sin protecciones» $y$ «no regulado por el Estado» (Poster y Salime, 2002), vemos cómo el trabajo remunerado del hogar en Ecuador no encaja perfectamente con esta definición. Cómo describo más adelante, el trabajo del hogar ha recibido atención — si no regulación sistematizada - de parte del gobierno ecuatoriano, que ha declarado que las TRH tienen derechos laborales y merecen las mismas protecciones sociales que otros trabajadores. No obstante estas regulaciones son ampliamente ignorados, y la manera en que el trabajo se organiza es informal (Moreno Zúñiga, 2013; Toledo González, 2013). Este empleo combina la participación en el mercado laboral, vista como una actividad pública, con la ubicación en una casa privada haciendo quehaceres que en muchas casas no son remunerados (véase la línea sobre el trabajo del cuidado, por ejemplo, Carrasquer Oto, 2013; Gutiérrez-Rodríguez, 2014; Torns, 2008). El TRH ejemplifica, entonces, cómo «se van deshaciendo las dicotomías claras entre formal e informal» (Elson, 1999, p. 617). El primer convenio de la Organización Internacional del Trabajo (OIT) «enfocado a trabajadores y trabajadoras del sector informal» (Goldsmith, 2013), el Convenio 189 trata sobre los TRH y ha sido firmado por solo 22 naciones, indicando una falta de atención a nivel global.

En general, los ministerios del trabajo latinoamericanos ponen poca atención a las TRH (la gran mayoría son mujeres, y por eso uso el marcador femenino). Política y políticos han mencionado lo que consideran la relación especial y delicada entre empleadores y empleadas del hogar, la cual puede ser perturbada por un aumento en los derechos legales. El estudio de los códigos laborales y las reformas a dichos códigos en Latinoamérica hecho por Carnes (citado en Blofield, 2012) provee una comparación útil. Muestra que en la región durante los 80 y 9o, mientras se implementaban reformas neoliberales, los códigos laborales no experimentaron rupturas, de hecho se incrementó la protección laboral. Pero las cláusulas discriminatorias a TRH no se reformaron. Entre 1980 y 2004 disminuyeron en cuatro países las máximas horas de trabajo legales por semana, después de las cuales se tendría que pagar horas extras. Ecuador bajó a 40 horas, Chile a 45, Venezuela y República Dominicana a 48. Sin embargo, este límite no aplicaba en muchos casos a los derechos legales de las TRH, que no son consideradas como iguales, sino como ajenas a este marco legal. Esto explica la débil relación entre las reformas de los códigos laborales y las reformas específicas en los códigos sobre trabajadores marginales como las TRH, quienes trabajan fuera del sector formal (Blofield, 2012). Hay que estudiar el trabajo remunerado del hogar como parte de la economía informal (Lautier, 2003), que es menos afectado que el empleo formal por regulaciones e intervenciones del Estado. 


\section{LA REPRODUCCIÓN SOCIAL DENTRO DEL CAPITALISMO}

El presidente ecuatoriano Rafael Correa celebra y proclama las virtudes del socialismo y el posneoliberalismo, pero la economía ecuatoriana es esencialmente capitalista; por tanto, la teoría marxista-feminista puede ser especialmente útil para explicar los sucesos económicos y laborales contemporáneos. En el sistema capitalista, el poder laboral se tiene que mantener y reproducir; extendiendo los argumentos de Marx, feministas marxistas han analizado el papel laboral (remunerado) de las mujeres de las clases trabajadoras, y también su «involucramiento en procesos que renuevan los productores directos»; es decir, procesos de reproducción social (Vogel, 2014, p. 129). Se ha criticado la sobrevaluación de la producción económica y la ignorancia de la reproducción social, que sostiene la producción atendiendo a las necesidades de los trabajadores productivos (Bryson, 2005). Las teorías de reproducción social frecuentemente se enfocan en el trabajo no remunerado que desempeñan las mujeres en la esfera doméstica (Benería, 2003; Giménez, 1990; MIES, 1998; Torns, 2008), pero el trabajo pagado en casas privadas también es parte de la reproducción social. Siendo una forma de reproducción social que genera remuneración, el empleo del hogar complica la división rígida entre producción y reproducción.

Las TRH están vendiendo su poder laboral, y sufren explotación por parte de sus empleadores, pero esta situación no corresponde con una visión típica de la relación entre capital y trabajo. Las actividades de las TRH, además, son iguales a las que desempeñan otras personas (en su gran mayoría, mujeres) sin percibir ningún ingreso (Carrasquer Oto, 2013). De hecho, las TRH ocupan el papel tradicional de la madre/esposa en la familia heterosexual: su trabajo en la casa hace posible el trabajo de otros fuera de la casa y apoya así la producción económica capitalista. El trabajo que antes le pasaba el hombre a su pareja mujer (sin pago), ahora se lo pasa esa mujer a otra mujer (con pago) cuando entra la empleadora al mercado laboral para participar de pleno en los procesos de producción. Todo este trabajo feminizado de reproducción social, incluso el cuidado de niños y adultos, se desvaloriza (Beneria, 2003; Carrasquer Oto, 2013; Pereyra, 2013). Las habilidades requeridas incluyen un rango de expresiones emocionales e íntimas (afectivas) que contribuyen al estereotipo del trabajo del hogar como femenino; esta labor emocional privilegia los sentimientos de la familia que emplea la TRH, reproduciendo la desigualdad de clase (Brites, 2014; Gutiérrez-Rodríguez, 2014).

Desde una perspectiva marxista, todo trabajo en relación de dependencia - trabajo hecho como empleado o empleada - es explotador. Sin embargo, el trabajo remunerado del hogar tiene particularidades que lo distinguen de otros tipos de empleo. Es un trabajo que no tiene «los estándares mínimos que hacen al trabajo decente» (Pereyra, 2013, citando a Valenzuela y Mora, 2009). El trabajo del hogar se destaca por una dominación personalizada, porque normalmente es una relación de empleo entre dos personas «con posiciones sociales asimétricas» (Toledo González, 2013; ver Borgeaud-Garciandía y Lautier, 2014; Casanova, 2013; Gorbán y Tizziani, 2014; León, 2013). En este contexto de desigualdad íntima, incluso la comida se vuelve un territorio de conflicto entre las personas de distintas clases sociales, parte de un fenómeno que he llamado la «inequidad encarnada» (Casanova, 2013; Gorbán, 2013; Saldaña-Tejeda, 2013). Bruno Lautier (2003) ha sugerido que es justo por esta desigualdad y por la forma en que se 
benefician las élites de ella, los académicos han evitado estudiar la situación de las TRH —no tienen la distancia suficiente, y sus hallazgos pueden amenazar los privilegios que disfrutan como empleadores de TRH- La particularidad del trabajo remunerado del hogar se refleja en la ley y en los códigos laborales, que históricamente y hoy en día, o excluyen a las TRH, o les otorgan menos derechos que otros trabajadores (Abrantes, 2013; Goldsmith, 2013; Maich, 2014; Pereyra, 2013). En su descipción del encuentro internacional que produjo el Convenio 189 de la oIT, Mary Goldsmith (2013) relata que:

Los empleadores y, en menor grado, algunos gobiernos enfatizaban que no se podían equiparar completamente los derechos de las trabajadoras domésticas con los del resto de los trabajadores, por tratarse de una actividad que se desarrolla en un hogar para una familia. (p. 241)

Este discurso político reafirma la división entre el trabajo productivo y reproductivo, simbólicamente excluyendo a las TRH de la fuerza laboral, tanto como al nivel micro lo hacen los discursos «es como parte de la familia» o «es una ayuda» para oscurecer la relación laboral (Moreno Zúñiga, 2013). Simbólicamente subordina el trabajo de reproducción social al trabajo productivo, una jerarquía que tiene implicaciones para el trabajo informal, el trabajo del cuidado, y el trabajo no remunerado, comúnmente desempeñados por las mujeres.

\section{UNA REALIDAD LOCAL COMO ESTUDIO DE CASO}

Guayaquil es la urbe más grande del Ecuador, con casi dos y medio millones residiendo en la ciudad y unos tres millones en el área metropolitana (M. I. Municipalidad de Guayaquil, 2016). Muchos guayaquileños viven en la pobreza; según Floro y Messier (2006), el 87\% era pobre al comienzo de los 2000; según el gobierno actual, la pobreza se ha reducido a un $10 \mathrm{u}$ $11 \%$, aunque hay dudas acerca de esta figura, ya que generalmente las familias de clase media y alta emplean TRH, y para muchas de estas familias, la única señal concreta de que sí son de clase media es la presencia de una TRH en su casa.

El período escogido (2010-2015) para esta investigación sobre el TRH en Guayaquil fue ideal. En 2009, el gobierno de Correa había comenzado una campaña para hacer respetar las leyes laborales que protegían a las trabajadoras del hogar, pero no hubo una implementación de forma sistemática o sostenida. Desde esas fechas, la situación de las TRH aparecía con más frecuencia en las noticias, y las organizaciones que apoyaban a estas trabajadoras incrementaron sus esfuerzos educativos y publicitarios para aprovechar el nuevo interés de parte del gobierno, con la meta de mejorar las condiciones del trabajo. En 2010, cuando se empezó este trabajo de campo, funcionarios del Ministerio de Trabajo iban de casa en casa en los barrios de clase alta de Guayaquil, haciendo inspecciones para determinar si había TRH y si éstas estaban recibiendo el salario mínimo - entonces era de USD 240- y los beneficios mandados por el gobierno, como, por ejemplo, la afiliación al seguro social. De acuerdo con miembros («socias») de la organización estudiada, los empleadores, que se habían enterado de la presencia de los inspectores en su vecindario, solían borrar la evidencia física de la TRH por varios métodos: dándole un día libre, escondiéndola en otra parte de la casa, diciendo que era una prima del campo que estaba de visita. En 2012 este escenario había cambiado ya, se habían 
terminado las inspecciones, aunque las clases medias y élites todavía se sienten amenazadas por la retórica populista-socialista del gobierno. Lo paradójico es que la luz pública sobre el tema de los derechos de las TRH en este período dificultó aún más la situación de algunas TRH. Empleadores temían ser investigados o demandados y las trabajadoras temían ser despedidas — si ya tenían trabajo- o no obtener empleo — si estaban buscándolo-.

La organización objeto de este estudio de caso — que aquí se denominará Organización de Trabajadoras del Hogar (отн) - se inició en Guayaquil a finales de los años noventa. La ОTH colabora con otras organizaciones sin fines de lucro y organizaciones no gubernamentales (ONG), y disfruta de relaciones amistosas con algunos sindicatos. Tal vez como producto de su participación en cumbres y redes internacionales, la OTH se enfocó por varios años en presionar al gobierno ecuatoriano para la firma del Convenio 189 de la OІт. Este esfuerzo fue exitoso: Ecuador ratificó el convenio en 2013. Las actividades de la Отн incluyen: asesoría legal gratuita para TRH cuyos derechos han sido violados por sus empleadores; talleres sobre derechos laborales y otros temas (violencia doméstica, autoestima); campañas de concientización dirigidas a empleadores y al público; manifestaciones en sitios locales como el Ministerio del Trabajo y las oficinas del Seguro Social (IESS). La OTH difunde información sobre los derechos que las TRH ya tienen bajo la ley: afiliación al sistema nacional de seguridad social (con aportes de empleadores); vacaciones y licencia por maternidad; pago por horas extras; indemnización por despido; un buen ambiente de trabajo; no ser discriminadas. La отн no tiene una lista de miembros ni un sistema estructurado de afiliación al grupo, pero hay un núcleo activo de unas 20 a 30 mujeres. En su mayoría, son ex-TRH, aunque participan también algunas que actualmente trabajan como TRH. La organización recibe fondos principalmente de ONG con base en Europa.

Para este estudio de caso se usaron varios métodos de recolección de datos: observación participante, análisis de documentos (anuncios clasificados, noticias en la prensa) y entrevistas. En 2010, 2012, 2014, y 2015 se realizó una observación participante con la OTH y sus socias en Guayaquil dentro y fuera de la oficina, asistiendo a reuniones formales e informales, talleres educativos, ruedas de prensa, eventos sociales (bingos), manifestaciones y plantones. Se hizo una entrevista formal a $14 \mathrm{TRH}$ - ex, actuales y desempleadas - que participaban en las actividades de la отн y también se entrevistó a tres empleadores de TRH (Casanova, 2013). Ampliando estos métodos para profundizar más en el tema, en 2014 se hizo una investigación cuantitativa a base de encuestas, colaborando con la OTH para recopilar datos de 400 TRH actuales y ex (Casanova, Rodríguez Soto, y Bueno Roldán, 2015). Este trabajo comparte con la socióloga Rebeca Moreno Zúñiga sobre la utilidad de un estudio de caso para examinar el trabajo del hogar: «El estudio de caso consiste en una descripción detallada de una situación en particular o de los actores que confluyen en ella para comprender sus acciones en circunstancias concretas» (2013, p. 92). No se pueden sacar conclusiones generales en base a un estudio de caso, pero se puede profundizar en la realidad social de un lugar. Este estudio de caso es significativo también porque con pocas excepciones, (Casanova, 2013; Radcliffe, 1999), Ecuador no ha sido representado en la literatura sobre el trabajo remunerado del hogar.

Las trabajadoras que han intervenido y han estado alrededor de la Отн no se identifican usando el vocablo que prefieren los ecuatorianos de clase media: empleadas. Tampoco les gusta la palabra domésticas. En frase inolvidable de una de las participantes en esta investigación: no 
es adecuada la palabra doméstica porque «no somos domesticadas». Prefieren el término trabajadoras remuneradas del hogar, así que así se lo ha usado en este estudio. Se usa el sustantivo femenino trabajadora para referirse a las personas que trabajan en casas, que en su gran mayoría son mujeres. Se usa empleadores para referirse a hombres y mujeres que emplean a las TRH.

\section{EL RETO DE LA DIVULGACIÓN DE INFORMACIÓN, DEL TRABAJO DE CAMPO Y DE LA EDUCACIÓN POPULAR}

La OTH enfrenta continuamente retos en sus esfuerzos para mejorar las condiciones de trabajo de las TRH. Muchas de las actividades de la отн intentan, por ejemplo, concienciar a las trabajadoras sobre sus derechos, organizarlas y movilizarlas para defender esos derechos, de involucrarlas en la vida de la organización. Estas actividades son imprescindibles y son unas de las principales razones por la cual existe la оTH, como explica la presidenta del grupo:

Nosotras, las que estamos en este camino que seguimos, tratamos de formar activistas o promotoras en derechos laborales y seguridad social, [luchar por la] valoración del trabajo del hogar y poder mandarlas a las compañeras a hacer todo este tipo de réplicas [...] directamente a los sectores. Si es de hacerlo de casa en casa, de puerta a puerta como se dice, mucho mejor, [...] para que las compañeras conozcan sus derechos y tratar de incentivarlas [a participar en la organización] porque ése es otro problema.

Muchas organizaciones tratan de construir la base de movilización comenzando con actividades educativas y servicios a la gente que quiere movilizar (Léon, 2013). Sin embargo, este trabajo de campo, de ir de puerta en puerta, es difícil por la población de trabajadoras que vive en las casas de los empleadores - modalidad denominada puertas adentro-y por las largas jornadas de trabajo de las TRH. Las trabajadoras puertas adentro muchas veces son migrantes de provincia y carecen de tiempo y oportunidades para hacer conexiones con gente fuera de la casa en la que trabajan. La línea de investigación sobre el aislamiento de las trabajadoras puertas adentro (Borgeaud-Garciandía y Lautier, 2014; Lan, 2006; Lautier, 2003; León, 2013), cuenta sobre estas desventajas aunque también sabemos que en Latinoamérica las tasas de TRH puertas adentro están bajando rápidamente (Brites et al., 2013; Brites, 2013; Moreno Zúñiga, 2013). Las TRH que conviven con los empleadores tienden a trabajar más horas y tener menos vacaciones que trabajadoras que no conviven con los empleadores (puertas afuera).

En Guayaquil, la mayoría de las trabajadoras puertas afuera viven en lugares mal servidos por el transporte público, así que tienen un viaje bastante largo de la casa al trabajo. Tienen largas jornadas y muchas veces trabajan seis días de la semana. Muchas de las TRH a quienes se quisiera organizar viven en barrios marginales y de bajos recursos en el sector suroeste de Guayaquil llamado Isla Trinitaria. Es un área algo separada de la ciudad y sus barrios más establecidos. Las que residen ahí a veces tienen que viajar hasta dos horas para llegar a sus trabajos. Las TRH tienden a ser madres (y muchas, madres solteras), y su tiempo libre, los domingos, lo pasan con sus familias, descansando o poniéndose al día con los quehaceres de la casa y los asuntos de los niños, cosas que no pueden hacer entre semana. Varias trabajadoras entrevistadas se preocupaban de no poder pasar tiempo suficiente con sus hijos; 
cualquier rato que pueden compartir es valioso. El único día que las de la отн saben que las trabajadoras están en su propia casa y se las podría contactar, es el día que menos van a querer abandonar a la familia para participar en actividades que las llevan lejos de la casa y no les pagan nada.

A diferencia de trabajadores o trabajadoras en fábricas o en oficinas, muchas TRH no se ven cuando llegan al trabajo en la mañana, y no se encuentran durante la hora del almuerzo o en eventos sociales dentro o cerca del trabajo. Su trabajo es invisible — no sólo para el público-, sino también para las otras TRH, quienes trabajan en aislamiento en otros espacios privados por toda la cuidad (sobre la invisibilidad del trabajo del hogar, ver Borgeaud-Garciandía y Lautier, 2014; Brites, Tizziani, y Gorbán, 2013; Gutiérrez-Rodríguez, 2014; Lautier, 2003; Pereyra, 2013). $\mathrm{Al}$ ser cuestionadas si trataban con otras TRH cerca de las casas donde trabajaban, la mayoría de las trabajadoras entrevistadas afirmaban que eso no pasaba. El reto para la отн y organizaciones similares (León, 2013) es buscar las formas de alcanzar, educar, involucrar y, en última instancia, movilizar a trabajadoras que pasan casi todo el día haciendo un trabajo físicamente agotador (Casanova, 2013), solitario y socialmente invisible. Éste es un reto que también enfrentan otros grupos que buscan organizar trabajadores informales y precarios (Kabeer, Sudarshan; Milward, 2013). Los espacios donde ocurre el trabajo remunerado del hogar son tan múltiples y fragmentados que incluso las vendedoras del mercado o los trabajadores agrícolas responderían de mejor manera a las tácticas de movilización y organización tradicionales que una TRH. En parte, la falta de movilización masiva en este sector de empleo tiene que ver con este reto logístico: no se puede persuadir a las trabajadoras a participar en acciones colectivas si no se puede físicamente localizarlas y alcanzarlas. Algunos de estos retos han sido mencionados en estudios recientes sobre la movilización de trabajadoras domésticas en Brasil y en la India, pero parece que en estos casos existían otros factores que ayudaron a las organizaciones, como por ejemplo el uso de los colegios nocturnos como lugares de reclutamiento, vínculos con sindicatos poderosos u otros movimientos sociales, y el establecimiento de una agencia de empleo (Bernardino-Costa, 2014; Cornwall et al., 2013; Menon, 2013).

\section{EL RETO DE HACER CUMPLIR LAS LEYES}

Dado que predominan arreglos de empleo informales en vez de contratos escritos (Moreno Zúñiga, 2013; Pereyra, 2013), y la invisibilidad del trabajo en casas privadas (Brites et al., 2013), hacer cumplir las leyes laborales que existen para proteger a las trabajadoras remuneradas del hogar es una tarea casi imposible. En una ciudad tan grande como Guayaquil fue impracticable hacer una inspección exhaustiva de casa en casa para identificar a TRH y determinar si sus derechos legales eran reconocidos por los empleadores. Se supondría que la inspección conduciría a un proceso de seguimiento o unas consecuencias concretas para los que no estaban cumpliendo con las normas laborales, pero al parecer no se produjo semejante resultado, según lo manifestado por los participantes en esta investigación.

No obstante, según los resultados de una encuesta hecha en 2014 (Casanova et al., 2015) y los relatos anecdóticos de socias de la отн, empleadores y otros ecuatorianos, los derechos de las TRH se están respetando más que en el pasado. Más TRH ahora están afiliadas al IEss, lo 
que les proporciona cuidado de salud, pensión/jubilación y otros beneficios. Según reportes de los medios de comunicación, la afiliación de trabajadoras domésticas en Guayaquil se ha incrementado, de $8 \%$ en 2005 a 12,5\% en junio del 2010 y $20 \%$ en diciembre del 2010 (Expreso, 2005; El Universo, 2010a; El Universo, 2010b). Una encuesta conducida en 2014 con una muestra de TRH encontró una tasa de afiliación de 56\% (Paspuel, 2014) y la encuesta del mismo año de este trabajo reportó que menos de $20 \%$ estaban afiliadas (Casanova et al., 2015); de manera que es debatible el incremento exacto de la afiliación en este sector. En todo caso, parece que sí han aumentado las afiliaciones en la última década. Algo de este aumento resulta de los empleadores que afilian a trabajadoras que ya estaban trabajando en sus casas; pero sin duda, también resulta de los nuevos acuerdos entre empleadores y TRH que ahora entablan una relación laboral conscientes de que la afiliación es un requisito legal.

Hay casos de TRH — algunas de ellas asociadas con la оTH - que han demandado a sus exempleadores en el Ministerio de Trabajo pidiendo pagos atrasados, pago de horas extras e indemnizaciones. Cuando comenzó la observación participante con la ОTH en 2010, el grupo estaba monitoreando 13 casos legales de trabajadoras asociadas con la organización, con los nombres de las reclamantes y a veces el valor en dólares que se estaba exigiendo, todo ello listado en un cartel. El mero hecho de que estos trámites se habían iniciado y que estos casos están siendo atendidos por las agencias del Estado, es un indicador del reconocimiento legal de los derechos de las TrH. Aunque el proceso de demandar a exempleadores no ayuda a las TRH en el momento en que están trabajando, es una de las pocas formas de hacer valer la ley, aunque sea de forma retroactiva. No debemos asumir que la situación de las TRH ha cambiado completamente porque la encuesta revela que la mayoría no está gozando de todos los beneficios de ley, incluyendo el seguro social (Casanova et al., 2015).

El gobierno de Rafael Correa ha implementado nuevos programas sociales y económicos desde 2007 , cuando se instaló. Los esfuerzos del Estado para formalizar el trabajo remunerado del hogar y garantizar los derechos de estas trabajadoras parecían alcanzar su auge alrededor de 2010. Aunque hay cierta conciencia pública sobre el derecho de las TRH a ganar el sueldo básico (USD 366 en 2016), y aunque ha habido más demandas en contra de empleadores no cumplidores, existen límites a la ejecución de la ley. Estos límites resultan del hecho de que cada una es empleada por un hogar distinto; es decir, no hay un solo presidente de la compañía o una gerencia a donde puedan acudir colectivamente las trabajadoras. Otro reto, mencionado antes, es la invisibilidad del trabajo que se hace detrás de puertas cerradas en casas privadas. Incluso la idea tan sencilla de tener contratos escritos para las TRH, que también sería algo ventajoso para los empleadores, no ha sido adoptada en Guayaquil, tampoco en Buenos Aires ni Monterrey (Toledo González, 2013; Moreno Zúñiga, 2013). Aun teniendo ciertos derechos garantizados, las TRH siguen siendo trabajadoras precarias e informales. El problema de cómo hacer respetar las regulaciones y leyes que les otorgan derechos a las TRH ha sido mencionado por estudiosos del tema en América Latina (Blofield, 2012; Brites, 2013; Pereyra, 2013; Toledo González, 2013) y en Europa (Abrantes, 2013). Como han dicho las socias de la отн muchas veces - en relación a la aprobación de nuevas leyes (una meta importante)—, «el papel aguanta todo». Aprobar leyes es una cosa, y cambiar las condiciones de trabajo es otra. 


\section{EL RETO DE LA RELACIONES CON EL ESTADO Y LA REDEFINICIÓN DEL TRABAJO REMUNERADO DEL HOGAR}

Resulta necesario plantearse la relación entre la OTH como movimiento social y el gobierno ecuatoriano. Siguiendo la teoría de los movimientos sociales «nuevos» en Latinoamérica, se destaca el papel del gobierno nacional como una entidad a la cual pueden acudir los trabajadores y las trabajadoras para expresar sus preocupaciones (Escobar y Álvarez, 1992). Resalta también la capacidad de organizaciones como representantes de la clase trabajadora de reclamar al Estado y aplicar presión colectiva. El actual gobierno ecuatoriano, que al principio generó atención pública en cuanto a los asuntos de las TRH por medio de ruedas de prensa y campañas en los medios de comunicación, se ha vuelto un aliado con el cual no se puede contar. A pesar de que haber asistido a reuniones y eventos con el presidente hace un par de años, las líderes de la organización no han podido mantener la atención continua del Estado. El gobierno ha creado campañas sobre el trabajo remunerado del hogar (menos visibles que otras) y sin consultar con la оTH. En 2010, la presidenta de la organización afirmó:

El gobierno ha sido muy amplio con nosotras [después aclaró que no quería insinuar que la Отн hubiera recibido fondos del gobierno], todo esto del seguimiento y la que está haciendo la campaña del Ministerio de Trabajo es en base a la conversación que nosotros tuvimos un día con él [presidente Correa].

Apenas dos años después, ella y sus compañeras lamentaban no poder comunicarse con Rafael Correa ni con sus asistentes, y estaban recurriendo a enviarle correos electrónicos. También pusieron mensajes en su página de Facebook para tratar de llamar su atención y pedir una reunión donde podrían presentar sus reclamos y preocupaciones. Es poco claro por qué se había atenuado la relación antes estrecha entre la organización y el gobierno, y las líderes de la отн parecen estar algo confundidas por este cambio. Surgió una nueva apertura con la presentación pública en 2015 de los resultados de la encuesta que se hizo con la ОTH, que impulsó una serie de reuniones entre la organización y representantes del Ministerio de Trabajo y de la OIT, pero hay que ver si este contacto se mantiene y da frutos. Una perspectiva longitudinal nos ayuda entender la evolución de las relaciones entre el Estado y los movimientos sociales. Tal vez por esto es poco estudiado el aspecto político-organizativo del trabajo del hogar (Brites, 2013), con más estudios sobre otros temas como condiciones de trabajo, relaciones entre empleadores y TRH, migración internacional de TRH, (Casanova, 2013; Gorbán, 2013; Gorbán y Tizziani, 2014; Saldaña-Tejeda, 2015, 2013).

La отн, mientras lidia con estos retos, desempeña una gama de estrategias y actividades concretas, como la concientización dirigida a los empleadores, el establecimiento de contactos con organizaciones de TRH en otros países latinoamericanos, el trabajo en conjunto con sindicatos y ONG locales en proyectos de entrenamiento, y el apoyo de las luchas legales de las trabajadoras en contra de sus exempleadores. Aunque el movimiento de TRH en Ecuador es más pequeño que el movimiento de TRH en Brasil, se notan ciertas semejanzas, como el intento de aliarse con otros grupos progresivos (Bernardino-Costa, 2014) y el énfasis en ofrecer servicios como punto de entrada para TRH que después pueden unirse a las actividades políticas de la organización (Lautier, 2003). 
Considerando los retos enfrentados por la Отн, relacionados a la estructura y la invisibilidad del trabajo del hogar y su papel en la reproducción social — que lo excluye del trabajo productivo en el esquema capitalista-, la táctica más interesante empleada por estas líderes es la redefinición del trabajo remunerado del hogar como «trabajo regular» o como «cualquier otro trabajo». No solo las organizadoras más verbales y visibles, sino también las socias utilizan esta descripción. Un ejemplo de este discurso viene de una de las socias de la отн:

Parte de los principios es aplicar los derechos, para poder garantizar a las demás compañeras que todas tenemos derecho, como cualquier otro trabajador, como cualquier otra trabajadora.

La insistencia en reconocer el trabajo remunerado del hogar como trabajo, y la meta de «igualdad con otros trabajadores», es un discurso transnacional que surge de los encuentros internacionales de los movimientos de TRH, como el encuentro que produjo el Convenio 189 de la OIT (Goldsmith, 2013). En el folletín y otros materiales de la organización, solo se usa el sustantivo masculino trabajador en esta frase. Por lo tanto, la estrategia retórica para valorizar el trabajo remunerado del hogar de las mujeres lo compara con el trabajo pagado hecho por los hombres, que tiene menos estigma social. El trabajador ideal en este discurso es hombre, como también es el trabajador ideal en el pensamiento de los sindicatos locales (según las activistas de la отн). Usando más frecuentemente la forma masculina de la palabra, la отн no cuestiona, sino reproduce la asociación de los hombres con los conceptos de trabajo remunerado y de organización laboral o sindical (Borgeaud-Garciandía y Lautier, 2014). Simbólicamente acepta la división entre trabajo productivo y reproducción social.

Este discurse pone énfasis en el hecho de que la trabajadora del hogar es «una trabajadora» $\mathrm{y}$ «remunerada», no obstante la larga historia de «empleadas» $\mathrm{y}$ «muchachas» informales en los hogares ecuatorianos y de pago en especie. Una activista de la отн afirmaba que «las cosas están cambiando» como resultado de la nueva conciencia social y política de la situación de las TRH y ahora se puede exigir al empleador o empleadora «que se nos dé un seguro [social], que se nos dé un sueldo básico, que sea un trabajo igual que cualquier otro trabajo». Ahí estaba la frase frecuentemente usada por la отн: un trabajo igual que cualquier otro trabajo. Las que pertenecían a la organización y sus líderes repetían constantemente esta frase en conversaciones dentro del grupo, en entrevistas con periodistas y en acciones colectivas como protestas y ruedas de prensa.

\section{CONCLUSIONES}

Surge una contradicción en la estrategia de la отн de redefinir el trabajo remunerado del hogar como un trabajo regular. Los factores que conducen a la explotación excesiva de las TRH y dificultan la organización y movilización de las mismas no son las semejanzas del trabajo del hogar con otros tipos de trabajo, sino precisamente sus particularidades. Las condiciones específicas de la reproducción social no se pueden reducir a las demandas más comunes de los trabajadores en la producción capitalista. Estas particularidades, como mencioné antes, ponen a prueba la capacidad de la отн para llegar a las trabajadoras y la capacidad del gobierno para hacer valer o regular sus derechos. Declarar que las TRH son «iguales» que otros trabajadores 
- aunque este discurso ha sido usado por movimientos sociales en otros países (Cornwall et al., 2013, p. 151; Goldsmith, 2013) - no supera estos retos. La informalidad de la mayoría de los arreglos de empleo doméstico, que persiste aún en un momento de crecimiento de conciencia sobre los derechos de las trabajadoras del hogar, es muy difícil de cambiar. Las TRH son explotadas, mal pagadas, y se aprovechan de ellas por un sinnúmero de razones. Estas razones incluyen «la costumbre del lugar» — frase antes usada en la sección del Código de Trabajo ecuatoriano dedicada al trabajo doméstico-, la posición estructural de las trabajadoras como mujeres de bajos recursos — que pueden ser menores de edad y/o mujeres discriminadas por raza o color-, la invisibilidad de las trabajadoras porque laboran en espacios privados, la denigración de las actividades de reproducción social que desempeñan, y los ingresos moderados de los empleadores de clase media - que limitan su habilidad de pagar el sueldo mínimo y los beneficios-. Éste es un conjunto de condiciones que otros tipos de trabajadores no experimentan: condiciones relacionadas al bajo estatus de la reproducción social.

Cuando el trabajo remunerado del hogar se presenta como el equivalente de otros tipos de trabajo, la opresión y la explotación de las TRH se entiende como un encuentro típico entre capital y trabajo. Pero empleadores en hogares privados son distintos a los capitalistas arquetípicos - primero que nada, muchos no se ven como empleadores-, y usan técnicas distintas para controlar a las trabajadoras; técnicas más personalizadas, a menudo incorporando elementos de parternalismo/maternalismo (Borgeaud-Garciandía y Lautier, 2014) o de dominación racial, de género, y de clase (Brites, 2014; Gutiérrez-Rodríguez, 2014). Además, muchas TRH hacen trabajo de cuidado (Carrasquer Oto, 2013; England, 2005; Sarti, 2014; Torns, 2008), que requiere un nivel de compromiso emocional (Gutiérrez-Rodríguez, 2014) que no existe en otros tipos de empleo. Si los trabajadores pueden reclamar colectivamente al Estado por sus derechos, entonces las trabajadoras del hogar podrían sacar provecho de su inclusión con otros trabajadores «regulares». Pero el desajuste entre la insistencia en que las son iguales a otros trabajadores, y las condiciones únicas que sufren, afectan su experiencia en el trabajo y también son obstáculos para los grupos que tratan de organizar o concientizar a estas trabajadoras. Los movimientos de TRH en Brasil han tenido éxito con otra estrategia: resaltar las opresiones múltiples que les afectan a las TRH, usando un discurso «interseccional» que reconoce los aspectos de género, raza y clase que se combinan para explotar de forma única a estas mujeres (Bernardino-Costa, 2014). Para equiparar los derechos, no necesariamente hay que omitir las particularidades del trabajo del hogar.

Los hallazgos de este estudio de caso generan preguntas más amplias tanto para académicos como activistas: ¿Cuáles otros factores, aparte de las condiciones del trabajo en sí, que hacen posible o impiden la organización eficaz de trabajadores precarios como las TRH? ¿Sería posible sindicalizar a las trabajadoras remuneradas del hogar en Ecuador, y cuáles serían las ventajas y desventajas de tener sindicatos? ¿Cuáles desafíos específicos presenta el trabajo percibido como femenino y de reproducción social — como el trabajo del hogar- a las estrategias tradicionales de organización laboral? Considérese, además, que por el momento, hay obstáculos legales para la creación de sindicatos en vez de organizaciones de movimiento social; por ejemplo, un requisito de un número mínimo de empleados por empleador para formar un sindicato. Normalmente una TRH es la única empleada de sus empleadores. Éstas son preguntas 
que está enfrentando la отH en la actualidad y en un ambiente político donde las necesidades de las TRH son a la vez divulgadas por las ONG y algunas agencias del Estado y no tomadas en cuenta por los empleadores y la mayoría de los políticos.

Las mujeres trabajadoras remuneradas del hogar hacen labor reproductiva que beneficia a sus empleadores, en ocasiones a costa de sus propias familias. Esta transferencia de labor reproductiva diferencia el trabajo del hogar de otros empleos, incluso de otros empleos informales, a pesar de la estrategia retórica de presentarlo como cualquier otro trabajo. A las TRH ecuatorianas todavía no se les otorga las condiciones laborales y los beneficios garantizados por la ley y asociados con el trabajo productivo formal. Desde luego se debe seguir exigiendo derechos iguales para las TRH, pero se debería hacerlo de una forma que no ofusque las condiciones particulares de este empleo, sino que busque asignarle el valor merecido a la reproducción social; así, este proyecto tendría beneficio para muchas mujeres y no solo para las trabajadoras del hogar.

\section{REFERENCIAS}

Abrantes, M. (2013). A Matter of Decency? Persistent Tensions in the Regulation of Domestic Service. Revista de Estudios Sociales, (45), 110-122.

Bernardino-Costa, J. (2011). Destabilizing the National Hegemonic Narrative: the Decolonized Thought of Brazil's Domestic Workers' Unions. Latin American Perspectives, 38(178), 33-45.

Bernardino-Costa, J. (2014). Intersectionality and Female Domestic Workers' Unions in Brazil. Women's Studies International Forum, (46), 72-80.

Blofield, M. (2012). Care Work and Class: Domestic Workers' Struggle for Equal Rights in Latin America. Penn State University Press.

Borgeaud-Garciandía, N. y Lautier, B. (2014). La personalización de la relación de dominación laboral: las obreras de las maquilas y las empleadas domésticas en América Latina. Revista Mexicana de Sociología, 76(1), 89-113.

Brites, J. (2013). Trabajo doméstico en Brasil: transformaciones y continuidades de la precariedad. Trayectorias, 15(36), 3-19.

Brites, J. (2014). Domestic service, Affection, and Inequality: Elements of subalternity. Women's Studies International Forum, (46), 63-71.

Brites, J., Tizziani, A. y Gorbán, D. (2013). Trabajo doméstico remunerado: espacios y desafíos de la visibilidad social. Revista de Estudios Sociales, (45), 226-228.

Bryson, V. (2005). Production and Reproduction. En G. Blakely and V. Bryson (Ed.), Marx and Other Four-Letter Words (pp. 127-142). Ann Arbor: Pluto Press.

Cabezas Fernández, M. (2012). «10 años de lucha por la ley, 11 en el parlamento: las reivindicaciones de las trabajadoras asalariadas del hogar en Bolivia durante la etapa neoliberal. Íconos, (44), 85-10o.

Campaña para afiliarlas al IEss no se mantiene, dicen empleadas. (3 diciembre de 2010b). El Universo. Recuperado de http://www.eluniverso.com/2010/12/03/1/1445/campana-afiliarlas-iess-mantienedicen-empleadas.html.

Carrasquer Oto, P. (2013). El redescubrimiento del trabajo de los cuidados: algunas reflexiones desde la sociología. Cuadernos de Relaciones Laborales, 31(1), 91-113. 
Casanova, E. M. (2011). Making Up the Difference: Women, Beauty, and Direct Selling in Ecuador. Austin: University of Texas Press.

Casanova, E. M. (2013). Embodied Inequality: The Experience of Domestic Work inUrban Ecuador. Gender \& Society, 4(27), 561-585.

Casanova, E. M., Rodríguez Soto, L. y Bueno Roldán, R. (2015). Informadas pero inseguras: condiciones de empleo y protección social entre trabajadoras del hogar en Guayaquil, Ecuador. Manuscrito bajo consideración.

Chaney, E. M. y García Castro, M. (Eds.) (1991). Muchachas no more: Household Workers in Latin America and the Caribbean. Filadelfia, usA: Temple University Press.

Cornwall, A., Oliveira, C.M. y Gonçalves, T. (2013). If You Don’t See a Light in the Darkness, You Must Light a Fire. En N. Kabeer, R. Sudarshan, y K. Milward (Ed.), Organizing women workers in the informal economy: beyond weapons of the weak. London, England: Zed Books.

Ehrenreich, B. y Hochschild, A. R. (Eds.) (2002). Global woman: nannies, maids, and sex workers in the new economy. New York, UsA: Metropolitan Books.

El IESS sólo registra 4037 afiliados del servicio doméstico. (27 febrero de 2005). Expreso. Recuperado de www.explored. com.ec/wphoy-imprimir.php?id=198677

England, P. (2005). Emerging Theories of Care Work. Annual Review of Sociology, (31), 381-99.

Escobar, A. y Álvarez, S. E. (1992). The making of social movements in Latin America: identity, strategy, and democracy. Westview Press.

Estrada, D. (2009). Strides and Setbacks for Domestic and Rural Workers. Inter Press Service, (September 27). Recuperado de http://ipsnews.net/news.asp?idnews $=48613$.

Floro, M. y Messier, J. (2006). Tendencias y patrones de crédito entre hogares urbanos pobres en Ecuador. En G. Herrera (Ed.), La persistencia de la desigualdad: género, trabajo y pobreza en América Latina. Quito, Ecuador: FLACSO.

Giménez, M. E. (1990). The dialectics of waged and unwaged work: waged work, domestic labor, and household survival in the United States. En J. L. Collins y M. Giménez (Eds.), Work without wages: domestic labor and self-employment within capitalism (pp. 25-46). Albany, USA: State University of New York Press.

Goldsmith Connelly, M. (2006). Política, trabajo y género: la sindicalización de las y los trabajadores domésticos y el estado mexicano. En Orden social e identidad de género: México, siglos XIX-Xx (pp. 217-246). México DF, México: CiesAs.

Goldsmith, M. (2013). Los espacios internacionales de la participación política de las trabajadoras remuneradas del hogar. Revista de Estudios Sociales, (45), 233-246.

Gorbán, D. (2013). El trabajo doméstico se sienta a la mesa: la comida en la configuración de las relaciones entre empleadores y empleadas en la ciudad de Buenos Aires. Revista de Estudios Sociales, (45), 67-79.

Gorbán, D. y Tizziani, A. (2014). Inferiorization and deference: The construction of social hierarchies in the context of paid domestic labor. Women's Studies International Forum, (46), 54-62.

Gutiérrez-Rodríguez, E. (2014). Domestic work-affective labor: On feminization and the coloniality of labor. Women's Studies International Forum, (46), 45-53.

Hondagneu-Sotelo, P. (2007). Doméstica: Immigrant workers cleaning and caring in the shadows of influence. Berkeley, usa: University of California Press. 
INEC (2013). Encuesta nacional de empleo, desempleo y subempleo. Quito, Ecuador: INEC.

Jornada parcial, opción más acogida para las domésticas. (6 junio de 2010a). El Universo. Recuperado de http://www. eluniverso.com /2010/o6/o6/1/1445/jornada-parcial-opcion-mas-acogida-domesticas.html.

Kabeer, N., Sudarshan, R. y Milward, K. (Eds.) (2013). Organizing women workers in the informal economy. London, England: Zed Books.

Karides, M. (2002). Linking Local Efforts with Global Struggle: Trinidad's National Union of Domestic Employees. En N. Naples y M. Desai (Eds.), Women's activism and globalization: linking local struggles and transnational politics. New York, UsA: Routledge.

Lan, P. (2006). Global Cinderellas: migrant domestics and newly rich employers in Taiwan. Durham, UsA: Duke University Press.

Lautier, B. (2003). Las empleadas domésticas latinoamericanas y la sociología del trabajo: algunas observaciones acerca del caso brasileño. Revista Mexicana de Sociología, 65(4), 789-814.

León, M. (2013). Proyecto de investigación-acción: trabajo doméstico y servicio doméstico en Colombia. Revista de Estudios Sociales, (45): 198-211.

M. I. Municipalidad de Guayaquil (2016). Recuperado de http://www.guayaquil.gob.ec/

Maich, K. (2014). Marginalized struggles for legal reform: cross-country consequences of domestic worker organizing. Social Development Issues, 36(3), 73-91.

Menon, G. (2013). The Challenge of Organizing Domestic Workers in Bangalore: Caste, Gender and Employer-Employee Relations in the Informal Economy. En N. Kabeer, R. Sudarshan, y K. Milward (Eds.), Organizing women workers in the informal economy: beyond weapons of the weak. London, England: Zed Books.

Mies, M. (1998). Patriarchy and Accumulation on a World Scale. London, England: Zed Books.

Moreno Zúñiga, R. (2013). Las empleadoras del Área Metropolitana de Monterrey: interacciones sociales y acuerdos de contratación del servicio doméstico a tiempo parcial. Trayectorias, 15(37), 90-111.

Mose Brown, T. (2011). Raising Brooklyn: nannies, childcare, and Caribbeans creating community. New York, UsA: New York University Press.

OIT (Organización Internacional de Trabajo) [International Labor Organization (ilo)]. (2011). Coverage of domestic workers by key working conditions laws. Domestic Work Policy Brief, (5). Ginebra, Suiza: ILO.

OIT (Organización Internacional de Trabajo) [International Labor Organization (ilo)]. (2013). Women and men in the informal economy: a statistical picture. Second edition. Ginebra, Suiza: ILO.

Paspuel, W. (28 noviembre de 2014). El 44\% de las trabajadoras domésticas de Guayaquil no están afiliadas, según una encuesta. El Comercio. Recuperado de www.elcomercio.com/actualidad/trabajadoras-domesticas-guayaquil-afiliaci on-iess.html.

Pereyra, F. (2013). El acceso desigual a los derechos laborales en el servicio doméstico argentino: una aproximación desde la óptica de las empleadoras. Revista de Estudios Sociales, (45), 54-66.

Pérez, A. y Gallardo, C. (2005). Mujeres y hombres del Ecuador en cifras ii. Quito, Ecuador: conAmu.

Radcliffe, S. (1999). Race and domestic service: Migration and identity in Ecuador. En J. H. M. Momsen (Ed.), Gender, migration, and domestic service, New York, usa: Routledge.

Reyes Salazar, N. y Camacho Zambrano, G. (2001). Violencia contra las mujeres y los niños: situación del Ecuador (1995-1999). Quito, Ecuador: CONAMU. 